IEEE Industry Applications Society

Annual Meeting

New Orleans, Lousiana, October 5-9, 1997

\title{
An Adaptive, On-line, Statistical Method for Bearing Fault Detection Using Stator Current
}

\author{
Birsen Yazıcl, Gerald B. Kliman, William J. Premerlani, Rudolph A. Koegl, \\ Gregory B. Robinson, and Aiman Abdel-Malek \\ General Electric Company \\ Corporate Research and Development Center \\ Niskayuna, NY 12309 \\ Phone: (518) 3877247 Fax: (518) 3875975 E-mail: yazici@ crd.ge.com
}

\begin{abstract}
ilis wollknown that mobr current is a nonstationary signal whose propertes vary wih respoct the the varying cporating conditons of the motor. As a result Fourior analysis makes it cilicutt to recogniso faut conditions from the nomal cperating condtions of the motor. Thotrequency analysis, on the oher hand, unambiguously represents the motor cument which makes signal propertios relatod to fact debctonmore evident in the tansform domain $\boldsymbol{h}$ this paper, wo presentan adapive, statistical, tmotequancy mothod for the dotection of bearing futs. Dus to the time varying nomal operating conditions of the motor and the eflect of motor goomoty on tho current, we employ a training base approach in which the algoritm is trainod to recognito the nomal porating concitions of the motor botore the acteal bsting starts. The experimental resut's from our study suggests that the proposed mothod provides a poweril, and a general approach to the motor curentbased futtobtection.
\end{abstract}

\section{INTRODUCTION}

Motor current based fault detection relies on interpretation of the frequency components in current spectrum that are related to rotor asymmetries. However, the current spectrum is influenced by many factors, including electric supply, static and dynamic load conditions, noise, motor geometry, and fault conditions. Therefore, the motor current can be best modeled as a nonstationary random signal. Also, the dependency of motor current on the motor geometry requires a flexible, adaptive approach. For many years, the motor current analysis has been implemented using limited mathematical tools and computer capability. These methods are mostly deterministic and based on Fourier transformation [1]-[2]. However, it is well-known that Fourier transform techniques are not sufficient to represent nonstationary signals. Moreover, the uncertainty involved in the system requires an adaptive statistical framework to address the problem in an efficient way. In recent years, advancement of statistical signal processing methods have provided efficient and optimal tools to process nonstationary signals. In particular, time-frequency and time-scale transformations provide an optimal mathematical framework for the analysis of time varying, nonstationary signals [3]-[4].

It is well known that the frequency content of the motor current evolves over time as the operations conditions of the motor changes. This time evolution of nonstationary signals can be captured by the Gabor or short time Fourier transform [5]. This transform works by first dividing the signal into short consecutive or overlapping portions and then computing the Fourier transform of each portion. The idea is to introduce local frequency parameter so that the local Fourier transform looks at the signal through a window over which the signal is approximately stationary. Such a transform results in a two dimensional description of the signal in time-frequency plane composed of spectral characteristics depending on time.

The mathematical description of short-time Fourier transform is given as follows:

$$
F(\omega, n)=\frac{1}{\sqrt{2 \pi}} \int f(t) g\left(t-n t_{0}\right) e^{-i \omega t} d t
$$

where $g$ is an ideal cut-off function. Dividing the current signal into small portions amounts to multiplying $f$ by a translate of $g$, i.e., $g\left(t-n t_{0}\right)$ where $t_{0}$ is the length of the cut-off interval and $n$ is an integer associated with the signal portion. The parameter $\omega$ is similar to the 
Fourier frequency and many properties of Fourier transform carry over to the short time Fourier transform.

The Fourier analysis makes it difficult to recognize fault conditions from the normal operating conditions of the motor. Time-frequency analysis, on the other hand, unambiguous represents the motor current which makes signal properties related to parameter estimation, and recognition more evident in the transform domain. To illustrate this situation, we acquired motor current from a 3/4 HP motor operating under three different load condition. The motor was first operating under $2 \mathrm{lb}$-in of load, then $10 \mathrm{lb}$-in and then 2 $\mathrm{lb}$-in again. The analog signal was low pass filtered at $800 \mathrm{~Hz}$ and digitized at 32 samples per $60 \mathrm{~Hz}$ voltage supply. Time-frequency spectrum shown in Figure 1 clearly unveils the load oscillations which are important for fault detection. The horizontal axis represents the frequency with $0.2 \mathrm{~Hz} / \mathrm{bin}$ resolution and the vertical axis represents the time with 5 seconds resolution. The color represents the intensity of the time-frequency spectrum in $\mathrm{dB}$. The Fourier spectrum, on the other hand, will reveal no information about the load oscillations because, the time variations of the frequency content will be spread out over the entire frequency range.

In this paper, we propose an adaptive statistical timefrequency method to detect bearing faults. The block diagram of the proposed system is shown in Figure 2. The method consists of four stages: preprocessing, training, testing, and postprocessing. In the preprocessing stage, the analog data is subjected to typical signal conditioning procedures. During the training stage, the time-frequency spectrum of the current is computed and features relevant to fault conditions are extracted using torque and mechanical speed estimation. Next, the feature space is segmented into the normal operating conditions of the motor and a set of representatives and thresholds are determined for each normal operating mode. The segmentation is performed by either statistical segmentation or torque estimation. Once the algorithm is trained for all the normal operating conditions, the testing stage starts. In this stage, the time-frequency spectrum of the motor is acquired periodically and the features relevant to the fault conditions are extracted. Next the distance between the test feature and the representative of each normal operating condition is computed. If the test feature is beyond the threshold of all the normal operating conditions, it is tagged as a potential fault signal. During the postprocessing stage, the testing is repeated a number of times to improve the accuracy of the final decision.
The rest of the paper is organized as follows: In Section II, we introduce the preprocessing and training stages. In Section III-IV, we discuss the testing and postprocessing stages. In Section $V$, we present experimental results. Finally in Section VI, we briefly discuss further items of interest in this context and conclude our discussion.

\section{TRAINING}

In the preprocessing stage, the analog data is low pass filtered and digitized. Next, the time-frequency spectrum of the digital data is computed and fed into the training process. The first step in the training stage is the feature extraction process in which the frequencies relevant to fault detection are estimated from the timefrequency spectrum. Next, the training features are segmented into the constant operating modes of the motor. Finally, the samples from each mode are statistically analyzed to determine a mode representative and threshold. These values are saved in a database to be used as a baseline later in the testing stage. Note that the segmentation process and feature extraction process can be interchanged. However, the computational load is much less if the feature extraction is performed prior to segmentation because, the size of the feature vector is much smaller than the size of the entire frequency spectrum.

\section{A. Feature Extraction}

In this section, we shall discuss the estimation bearing fault frequencies. Next, we shall select a window of frequencies around the estimates to form a feature vector. A feature vector is a highly informative compressed data which facilitates reliable detection while reducing the computational load.

A bad ball bearing in a motor allows the shaft to move radially a small amount. For example, if there is a pit in the outer or the inner race, the balls encountering it will fall in and move radially. Thus the air gap geometry will be slightly disturbed leading to a modulation of the current. These modulation effects will show up at frequencies

$$
f_{\text {brg }}=f_{s} \pm n \cdot f_{v}, \quad n=1,2,3, \ldots
$$

where $f_{v}$ is the mechanical vibration frequency whose value depends on the type of the race defect and geometry of the bearing. If the bearings have six to twelve rolling elements, the inner race bearing defect frequencies can be estimated by [6]-[7] 


$$
f_{\text {brg }}=f_{s} \pm n \cdot 0.6 \cdot f_{m}, \quad n=6,7, \ldots, 12 .
$$

Similarly, the outer race bearing defect frequencies can be estimated by

$$
f_{\text {brg }}=f_{s} \pm n \cdot 0.4 \cdot f_{m}, \quad n=6,7, \ldots, 12 .
$$

A window of frequencies around the estimated bearing frequencies

$$
\begin{aligned}
& F_{b r g}(n)=\left[F_{b r g}^{6}(n), F_{b r g}^{7}(n), \ldots, F_{b r g}^{12}(n)\right] \\
& F_{b r g}^{n}(n)=\left[S\left(f_{b r g}^{n}-w, n\right), \ldots, S\left(f_{b r g}^{n}, n\right), \ldots, S\left(f_{b r g}^{n}+w, n\right)\right] \\
& n=6,7, \ldots, 12 .
\end{aligned}
$$

is selected to form the bearing feature vector where $S$ is the magnitude of the time-frequency transform. Typically, the length of the window is chosen to be at least $1 \mathrm{~Hz}$ on each side of the estimated frequency.

\section{B. Segmentation}

As discussed earlier, the motor current is a piecewise stationary signal in which different constant operating modes correspond to different statistically homogenous segments. Therefore, it is necessary to determine different baseline thresholds for different operating modes of the motor. To do so, we compute the timefrequency spectrum of the motor for all possible normal operating modes of the motor during the training stage, and segment the spectrum into homogenous segments along the time axis.

Let us assume a number of feature vectors $F(n)$, $n=1, \ldots, N$ are available for the training process where $N$ is the total training duration. We assume that the normal operating modes of the motor change slowly and contiguous feature vectors are likely to fall into the same mode. Given these assumptions, segmentation process can be viewed as detection of time instants $n_{s}$ at which $F(n), n \leq n_{s}$ and $F(n), n>n_{s}$ exhibit different statistical properties. We shall call this instant split point. To implement the detection process, we divide the feature vectors $F(n), \quad n=1, \ldots, N$ into contiguous windows along the time axis. The length of the time window $T$ is chosen so that the motor can exhibit at most two different operating modes during $T$ units of time. Within each time window, the location of the split point is detected by maximizing the conditional joint probability density function of the feature vectors within the window given the location of the split point. This can be mathematically expressed as $\hat{n}_{s}=\underset{n_{0}=0, \ldots, T-1}{\operatorname{Argmax}} \operatorname{Pr}\left(F\left(n_{0}+i\right), \quad i=0, \ldots, T-1 \quad \mid \quad i=n_{s}\right)$

where $n_{0}$ is the beginning of the time window, $n_{s}$ is the candidate location for the split point, and $F\left(n_{0}+i\right)$, $i=0, \ldots, T-1$ are the feature vectors within the time window. Note that $\hat{n}_{s}=T-1$ is interpreted as no mode change during the time window $T$. Once all the time windows are studied and the location of all the split points are estimated, segments that are similar are merged using the Bhattacharyya distance [8]. For more details on the probabilistic segmentation process, please refer to [9].

\section{Mode Representatives and Thresholds}

For each constant operating mode of the motor, the sample mean and the covariance matrix of the feature vectors are chosen as the representatives of the mode. Mathematically these representatives are given by

$$
\begin{aligned}
& M_{j}=\frac{1}{L_{j}} \sum_{k=1}^{L_{j}} F\left(n_{k}\right) \\
& C_{j}=\frac{1}{L_{j}} \sum_{k=1}^{L_{j}}\left(F\left(n_{k}\right)-M_{j}\right)\left(F\left(n_{k}\right)-M_{j}\right)^{T}, j=1, \ldots, L
\end{aligned}
$$

where $L$ is the total number of distinct operating modes of the motor, $L_{j}$ is the number of feature vectors belonging to the mode $j$, and $n_{k}$ is the time instance of a feature vector in a given homogenous mode. We shall refer the mean and covariance matrix of a mode as mode representative and denote it by

$$
R_{j} \equiv\left(M_{j}, C_{j}\right), \quad j=1, \ldots, L .
$$

It is important to note that once the bearing mode representatives are obtained, the distance between the distinct operating modes with respect to are calculated and stored in the database to be used in the postprocessing stage.

We now want to define the bearing fault thresholds for each normal operating mode of the motor. To do so, we first define a statistical distance between each member of the mode and its representative.

$$
\begin{aligned}
& D_{j, k}^{\text {brg }} \equiv d\left(F_{\text {brg }}\left(n_{k}\right), R_{j}^{\text {brg }}\right) \equiv \\
& \left(F_{\text {brg }}\left(n_{k}\right)-M_{j}^{\text {brg }}\right)^{T} C_{j}^{\text {brg }}{ }^{-1}\left(F_{\text {brg }}\left(n_{k}\right)-M_{j}^{b r g}\right) \\
& j=1, \ldots, L, \quad k=1, \ldots, L_{j} .
\end{aligned}
$$

Note that the distance measure is chosen to be the well-known Mahalanobis distance in which each entry in 
the Euclidean distance is down weighted by the variance of the entry [10].

In order to obtain an optimal bearing fault thresholds for each mode, the sample mean and standard deviation of the intra mode distances are calculated.

$$
\begin{aligned}
& \bar{D}_{j}^{\text {brg }}=\frac{1}{L_{j}} \sum_{k=1}^{L_{j}} D_{j, k}^{\text {brg }}, \\
& \sigma_{j}^{\text {brg }}=\sqrt{\frac{1}{L_{j}} \sum_{k=1}^{L_{j}}\left(D_{j, k}^{\text {brg }}-\bar{D}_{j}^{\text {brg }}\right)^{2}}, \quad j=1, \ldots, L .
\end{aligned}
$$

The bearing fault thresholds, $\tau_{j}^{\text {brg }}$, for each operating mode are set $\alpha$ unit standard deviation away from the mean distances, i.e.,

$$
\tau_{j}^{\text {brg }}=\bar{D}_{j}^{\text {brg }}+\alpha \sigma_{j}^{\text {brg }}, \quad j=1, \ldots, L .
$$

Note that in the case of normal distribution of the intra mode distances, $\alpha$ is typically chosen to be 2 to provide a $95 \%$ confidence interval. However in our approach, it is kept as an input parameter to allow the user to utilize one's engineering judgment.

\section{TESTING}

Similar to the training process, the test data is first subject to the operations in the preprocessing and feature extraction stage discussed above. Next, using the Mahanalobis distance introduced in Equation (2.7) the distance between the test feature $F_{t}$ and the representatives of the normal operating modes $R_{i}, \mathrm{~s}$ computed.

$$
\Delta_{j}^{b r g}=d\left(F_{t}^{b r g}, R_{j}^{b r g}\right), \quad j=1, \ldots, L .
$$

Next, we check if the distance $\Delta_{l}^{b r g}$ between the test feature and the representatives of each mode is less than the mode threshold $\tau_{j}^{b r g}$. If the distance is greater than all the mode thresholds, $\tau_{j}^{b r g}, j=1, \ldots, L$, then the test measurement is tagged as potentially faulty.

To improve the accuracy of our decision making, we repeat this testing process for multiple test features. The decisions obtained from the test features and the distances are fed to the postprocessor to finalize the decision on the bearings of the motor.

\section{POSTPROCESSING}

The assumption in the postprocessing stage is that, if there is a bearing fault, consecutive test samples are expected to be potential fault features and will eventually form a mode which is statistically distinct from all the normal operating modes of the motor. Therefore as the potential fault features are identified by the testing stage, the mean, $M_{F}$, and the covariance matrix, $C_{F}$, of the potential fault features are computed. Next, the distance, $B\left(R_{F}, R_{j}\right)$, between the normal operating modes of the motor and the representatives, $\left(M_{F}, C_{F}\right)$ of the faulty features are computed using the Bhattacharyya distance. The normal operating mode to which the potential fault representative is closest is identified, i.e.,

$$
\hat{j}=\underset{j=W_{L}}{\operatorname{Argmin}} B\left(R_{F}, R_{j}\right)
$$

The shortest Bhattacharyya distance, $\Omega_{\hat{\gamma}}$, of the mode $\hat{j}$ is retrieved from the database and compared with the $B\left(R_{F}, R_{j}\right)$. If the distance $B\left(R_{F}, R_{\hat{j}}\right)$ is greater than the distance $\Omega_{j}$, the final alarm for the faulty bearings is triggered.

\section{EXPERIMENTAL RESULTS}

For bearing fault detection experiments, a $3 / 4 \mathrm{HP}$ motor was used. The analog current data was low pass filtered at $800 \mathrm{~Hz}$, and digitized at 32 samples per power cycle. However, as we shall discuss, the algorithm does not require frequencies larger than 300 $\mathrm{Hz}$, and sampling frequency can be as low as 6 samples per power cycle. Each data file in the experiments contains 8 channels which includes 3 phase currents, 3 phase voltages, $60 \mathrm{~Hz}$ notch filtered first phase current, and accelerometer data. Notch filtered data was collected in anticipation of improving the dynamic range of the A/D converter. Accelerometer data was used to validate the location of the fault frequencies.

The experiments were performed using the 1st, 6 th, and the 8th channels to evaluate the significance of notch filtering, and the accelerometer data. The data was collected for about 40 seconds yielding 80000 points per channel. Later, these data sets were down sampled to study the effect of lower rate sampling. The length of the windowing function was selected so that the timefrequency spectrum has $0.2 \mathrm{~Hz} / \mathrm{bin}$ resolution. which resulted in 8 contiguous frequency spectra for each data set.

Table 1 shows the data sets used during the training stage of the bearing fault detection algorithm. The data was collected at different loads and speed. The bearing $\mathrm{C} 2$ is a healthy ball bearing which was pressed on the motor shaft, and then soaked with oil before it was installed. The motor was run continuously for about 5 minutes during the various tests. In one case, an 
artificial turn fault was induced to check if the algorithm would be able to distinguish between the turn faults and bearing faults. Note that for the healthy modes non zero label is used, the label 0 is reserved for the fault modes.

Table 2 shows the data sets acquired from the same motor with faulty bearings. The inner and outer race defects were simulated by mounting the faulty bearing in different configurations. In the first case, the ball bearing with a hole mounted towards the outer part of the rotor shaft inducing a severe outer race defect. In the second case, the bearing mounted towards the inner part of the rotor shaft inducing a mild outer race defect. Figure 3 illustrates the time-frequency spectrum of the motor current with healthy bearings around the bearing frequency. Ideally, the estimated frequency has to be at the middle of the window. However, the true bearing frequency usually shows up 2 to 5 bins off the estimated frequency. Figure 4 illustrates the timefrequency spectrum of the same motor with faulty bearings.

During the testing process, data from both Table 1 and Table 2 were used. The training data was included in testing to validate the threshold selection criteria. The threshold was set as 2 unit standard deviations. Out of 112 samples from the 6th channel, 110 samples were correctly identified. 2 samples from a normal operating mode with healthy bearings were misclassified as potential fault signals, yielding $98 \%$ accuracy and $2 \%$ false positive error. Samples from a normal operating mode were mostly within two standard deviation of the representatives. The false positive error is produced by those samples which are beyond two standard deviations. Nevertheless, these samples were still within at most 3.5 standard deviations of the representatives, which is very small as compared to the distance of even the closest fault signal. The detailed results of this test are tabulated in Table 3 . The diagonal entries in the matrix show the number of correctly classified samples and the off diagonal entries show the number of misclassifications. For example, the entry at the ith row and jth column shows the number of samples from mode i which are classified as mode j. In case of perfect classification, the matrix becomes diagonal. The average distance of the measurements from faulty bearings to the normal operating modes are at least in the order of hundred unit standard deviations. Therefore, the misclassifications can be easily avoided by analyzing the threshold setting further which was arbitrarily selected as two unit standard deviations.

The experiments with the unfiltered data yielded the same result as the filtered data. In fact, visual inspection of the feature vectors from both data sets did not reveal any significant difference. The tests were repeated with the data down sampled to 12 samples per power cycle. The notch filtered data resulted in $95 \%$ accuracy and $5 \%$ false positive rate. The unfiltered data resulted in $93 \%$ accuracy and $7 \%$ false positive rate. These results suggest that the proposed method does not require a high sampling rates.

Bearing experiments were also carried out with defective bearings of 1,2 , and 3 scratches on the outer race, 1,2 , and 3 scratches on the balls, and 1,2 , and 3 cage defects. All the defective measurements were correctly classified as defective.

\section{CONCLUSIONS}

In this paper, we discussed an adaptive time-frequency method to detect bearing faults. It was shown that the time-frequency spectrum reveals the properties relevant to fault detection better than the Fourier spectrum in the transform domain. The method is based on a training approach in which all the distinct normal operating modes of the motor are learned before the actual testing starts. Our study suggests that segmenting the data into homogenous normal operating modes is necessary, because, different operating modes exhibit different statistical properties due to nonstationary nature of the motor current. Overlooking at this fact will deteriorate the performance of the detection. We showed that signals from faulty motors are several hundred standard deviation away from the normal operating modes which indicates the power of the proposed statistical approach.

Our study suggests that the proposed method is a mathematically general and powerful which can be utilized to detect any fault that could show up in the motor current.

\section{REFERENCES}

[1] G.B. Kliman and J. Stein, "Methods of motor current signature analysis," Electric Machines and Power System, pp. 463-474, 1992.

[2] G.B. Kliman, The Detection of Broken Bars in Motors, EPRI GS-6589-L, Final Report, January 1990.

[3] J. Bertrand and P. Bertrand, "Time-frequency representation of broad band signals," pp. 164-171 in Combes, Grossman, and Tchamitchian, 1989.

[4] B. Boashash, Time-frequency signal Analysis, in Advances in Spectrum Analysis and Array Processing, S. Haykin, Prentice-Hall, Englewood Cliffs, NJ, pp. 418$517,1990$. 
[5] D. Gabor, "Theory of communications," Journal of IEE (London), Vol. 93, pp. 429-457, 1946.

[6] R.R. Schoen, T.G. Habetler, F. Kamran, and R.G. Bartheld "Motor bearing damage detection using stator current monitoring," in Cof. Rec. 29th Annual IEEE Ind. Applications Soc. Meeting, Oct. 1994, pp. 110-116.

[7] R.L. Schiltz, "Forcing frequency identification of rolling element bearings," Sound and Vibration. pp. 16-19, May 1990.
[8] An Introduction to Statistical Pattern Recognition, K. Fukunaga, Academic Press, 1990.

[9] B. Yazıcı and G.B. Kliman, "An unsupervised, on-line, method and apparatus for induction motor bearing fault detection using stator current monitoring," GE patent filed. Docket No. RD-24,3457. General Electric Company Corporate Research and Development Center, Niskayuna, NY, 1995.

[10] Pattern Recognition Principles, J.T. Tou, R.C. Gonzalez, Addison-Wesley Publishing Company 1974

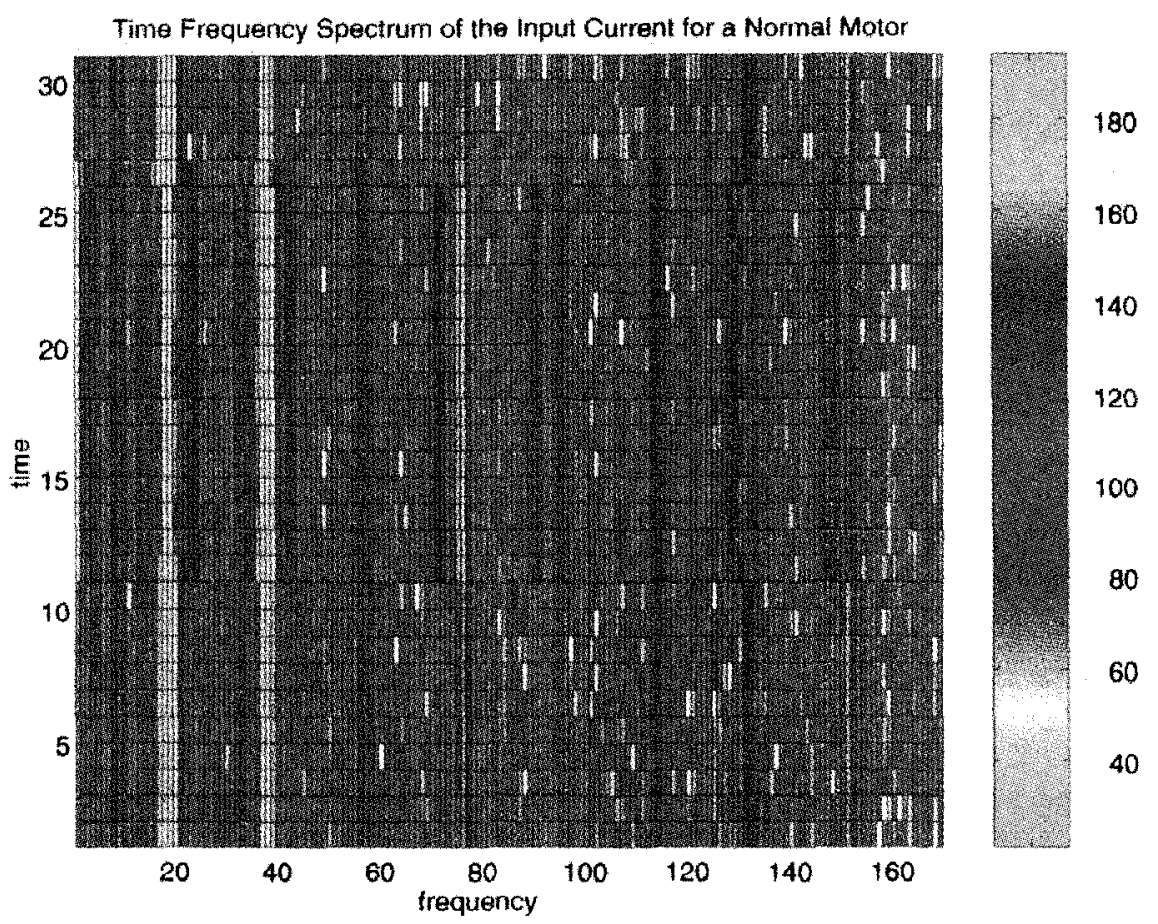

FIGURE 1

TIME-FREQUENCY SPECTRUM OF MOTOR CURRENT

Training Data

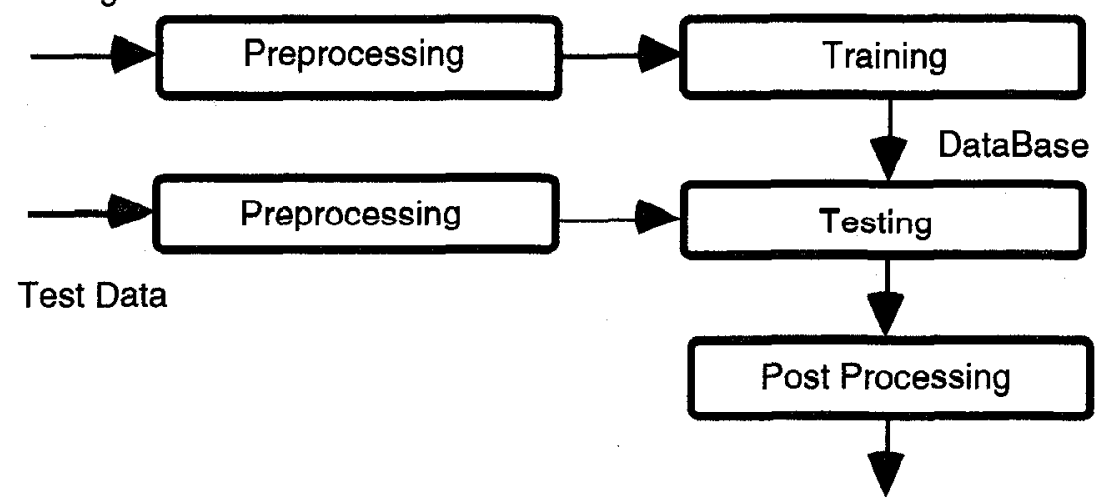

Decision

FIGURE 2

BLOCK DIAGRAM OF THE FAULT DETECTION METHOD 


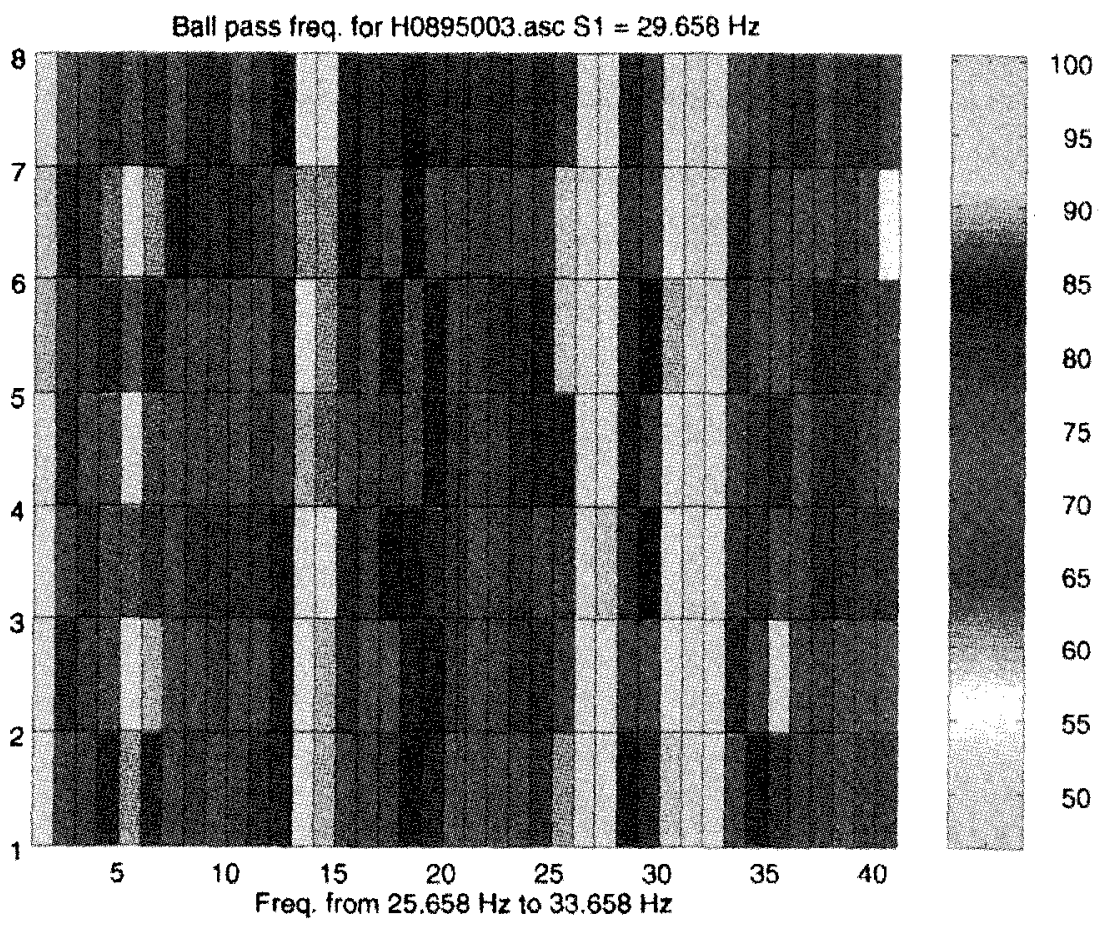

FIGURE 3

TIME-FREQUENCY SPECTRUM OF A NORMAL MODE

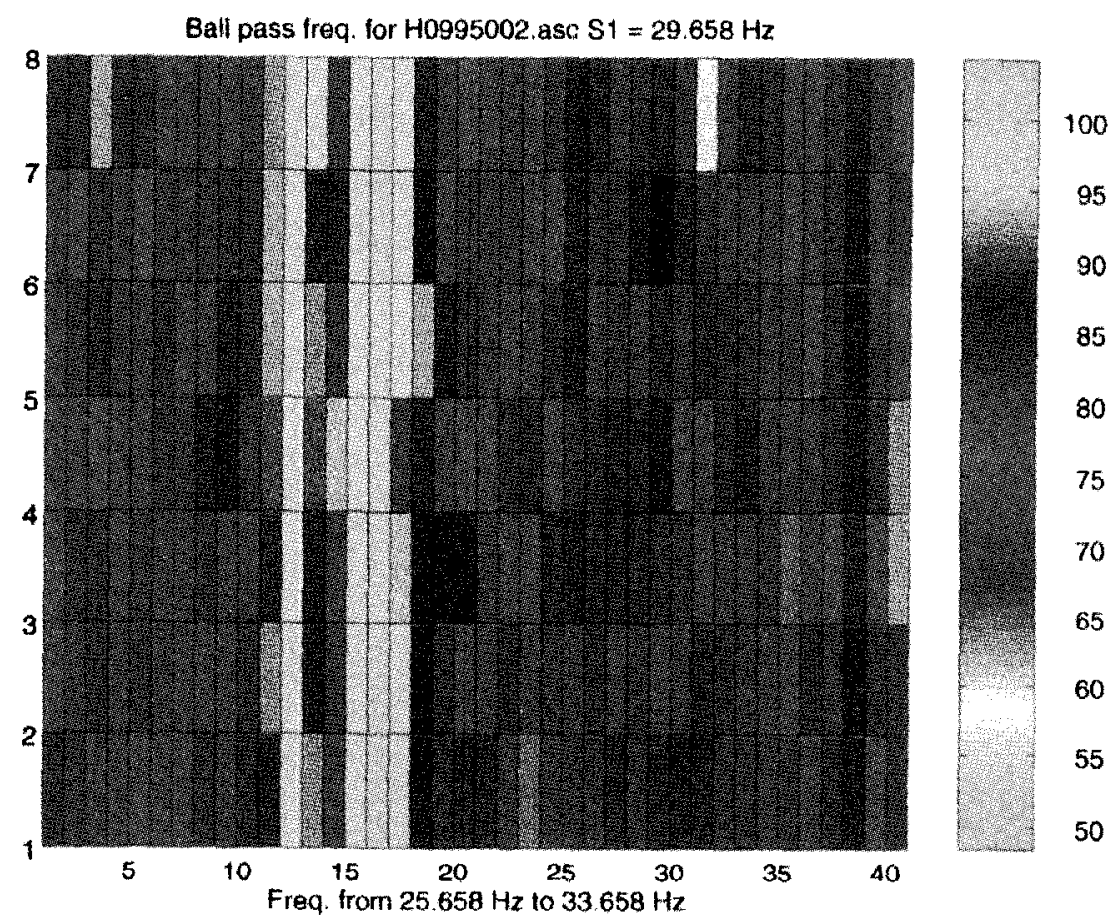

FIGURE 4

TIME-FREQUENCY SPECTRUM OF THE MOTOR WITH FAULTY BEARINGS 


\begin{tabular}{|l||l|l|l|l|}
\hline Mode & File Name & Torque & Spoed & Bearing and data ID \\
\hline 1 & H0895001.asc & $-3.00 \mathrm{lb}-$ in & $1789 \mathrm{Rpm}$ & $\mathrm{C} 2$ \\
\hline 2 & H0895002.asc & $+2.80 \mathrm{lb}-$ in & $1790 \mathrm{Rpm}$ & $\mathrm{C} 2$ \\
\hline 3 & H0895003.asc & $+12.00 \mathrm{lb}-$ in & $1762 \mathrm{Rpm}$ & $\mathrm{C} 2$ \\
\hline 4 & H0895004.asc & $+24.95 \mathrm{lb}-$ in & $1718 \mathrm{Rpm}$ & $\mathrm{C} 2$ \\
\hline 5 & H0895005.asc & $+12.00 \mathrm{lb}-$ in & $1762 \mathrm{Rpm}$ & C2 with turn faults \\
\hline
\end{tabular}

TABLE 1

DATA FROM 3/4 HP MOTOR WITH GOOD BEARINGS

\begin{tabular}{|c|c|c|c|c|}
\hline Mode & File Name & Torque & Spoed & Bearing and Data ID \\
\hline 0 & H0995001.asc & $+3.00 \mathrm{lb}$-in & $1789 \mathrm{Rpm}$ & H1 with hole out." \\
\hline 0 & H0995002.asc & $+12.20 \mathrm{lb}$-in & $1762 \mathrm{Rpm}$ & $\mathrm{H} 1$ with hole out. \\
\hline 0 & H0995003.asc & $+25.00 \mathrm{lb}-\mathrm{in}$ & $1720 \mathrm{Rpm}$ & $\mathrm{H} 1$ with hole out \\
\hline 0 & Ho995004.asc & $+12.15 \mathrm{lb}$-in & 1762 Rpm & $\mathrm{H} 1{ }^{*}$ hole out \& turn fault. \\
\hline 0 & H0995005.asc & $+2.85 \mathrm{lb}$-in & $1790 \mathrm{Rpm}$ & $\mathrm{H} 1$ with hole out. \\
\hline 0 & H0995006.asc & $+12.10 \mathrm{lb}$-in & $1762 \mathrm{Rpm}$ & H1 with hole out. \\
\hline 0 & H0995007.asc & $+25.00 \mathrm{lb}$-in & $1719 \mathrm{Rpm}$ & $\mathrm{H} 1$ with hole out. \\
\hline 0 & 11595001.asc & $+12.20 \mathrm{lb}$-in & $1761 \mathrm{Rpm}$ & $\mathrm{H} 1$ with hole in. ${ }^{* * *}$ \\
\hline 0 & 11595002.asc & $+25.65 \mathrm{lb}$-in & $1717 \mathrm{Rpm}$ & $\mathrm{H} 1$ with hole in. \\
\hline
\end{tabular}

TABLE 2

DATA FROM A 3/4 HP MOTOR WITH DAMAGED BEARINGS

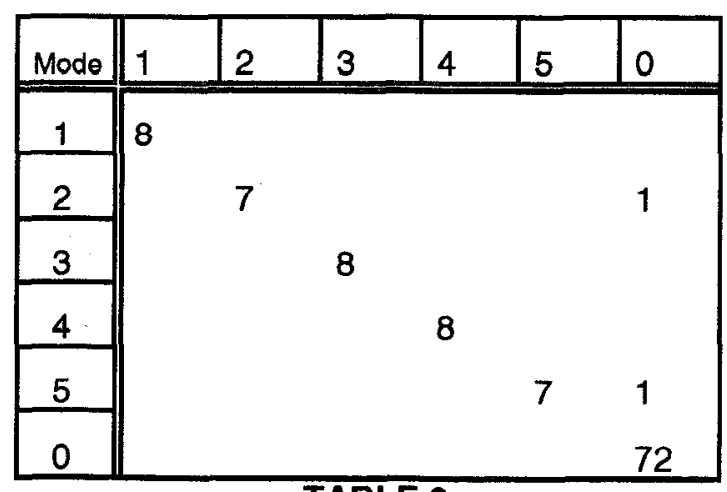

TABLE 3

RESULTS OF THE DETECTION TESTS FOR THE NOTCH FILTERED DATA SAMPLED AT 1923 HZ 